\title{
Physiological Mechanism of Genetic Law of Quantitative Characters
}

\author{
Huai Tang Gu \\ The Middle School of Dong-Xin Farm, Lianyungang City, Jiangsu Province, China. \\ E-mail: ght19651213@163.com
}

Received: December 4, 2019

Accepted: February 10, 2020

doi:10.5296/jbls.v11i1.16453

URL: https://doi.org/10.5296/jbls.v11i1.16453

\begin{abstract}
Regarding the genetic laws of quantitative characters, In 1908, Nilsson-EhleH. a Swedish geneticist, put forward the polygene hypothesis, and it makes a reasonable explanation for the genetic law of quantitative characters on the perspective of cytology. By using the law of conservation of mass and Le Châtelier's Principle, and proposed a point of view of a cyclical equilibrium system of chemical reactions to analyze and reason the physiological mechanism of genetic law of quantitative characters.
\end{abstract}

Keywords: quantitative characters, the law of conservation of mass, Le Châtelier's Principle, polygene hypothesis

\section{Introduction}

Quantitative character refers to the traits of continuous variation, regarding its genetic law, in 1908. Nilsson-EhleH., a Swedish geneticist, put forward the polygene hypothesis, (Liu Qingchang, 2009), he believed that quantitative characters are the result of the interaction of many independent genes, every gene has a little effect on the phenotypic expression, but its inheritance patterns still obeys the Mendel's genetic law, and assume that: 1. The effect of each gene was equal; 2. The alleles show incomplete dominance or no dominance, or increased or decreased efficiency; 3. The function of each gene can be cumulative. This hypothesis explains the genetic phenomenon of quantitative character on the point of view of cytology, then, what is its physiological mechanism? What is the chemical mechanisms that leads to the "micro effect" of quantitative character's genes? It is obviously worth for us to discuss further!

\section{Method}

\subsection{Expression of Quantitative Characters}

In genetics, traits refer to the morphology, physiological and biochemical property and the 
behaviors of organisms. The traits of a biological individual are expressed by genes that control protein's synthesis, which is completed after the process of transcription and translation. Quantitative characters are also expressed by genes encoding proteins, we call this protein, which is synthesized by gene coding and it can lead to expression of quantitative characters, expressive substance of quantitative character, or expressive substance (A).

\subsection{Cyclical Chemical Equation of Synthetic Expressive Substance of Quantitative Character}

The expressive substance (A) is transformed from another substance (B) after a series of physiological and biochemical reactions, namely anabolism, and the expressive substance (A) can be decomposed into (B) after a series of other chemical reactions, that is, catabolism, so as to form a cyclical chemical equation to synthesize the expressive substance of quantitative character, (that is, $\mathrm{B} \rightarrow \mathrm{C} \rightarrow \mathrm{D} \rightarrow \ldots \ldots \rightarrow \mathrm{A} \rightarrow \mathrm{E} \rightarrow \mathrm{F} \rightarrow \ldots \ldots \rightarrow \mathrm{B}$ ).

\subsection{Cyclical Equilibrium System of Chemical Reaction for Synthesis of the Expressive Substance of Quantitative Character}

Since the inheritance of quantitative characters are controlled by multiple genes, therefore, there are multiple cyclical chemical equations to synthesize expressive substance (A) in organisms, that is, there are multiple genes in the cell that simultaneously encode and synthesize the same protein that causes expression of quantitative characters. These cyclical chemical equations form a cyclic equilibrium system, which is centered on the expressive substance (A), for the synthesis of quantitative character expression substance (A).

\subsection{The Number of Reaction Factors and the Mass of Reactants in the Cyclic Equilibrium System of Chemical Reaction}

In a cyclical chemical equation, (that is, $\mathrm{B} \rightarrow \mathrm{C} \rightarrow \mathrm{D} \rightarrow \ldots \ldots \rightarrow \mathrm{A} \rightarrow \mathrm{E} \rightarrow \mathrm{F} \rightarrow \ldots \ldots \rightarrow \mathrm{B}$ ), because of the chemical equilibrium, the mass of every reaction factor is equal, namely, the mass of $\mathrm{B}$, C, D.....E, F, A is equal, set to (a). Therefore, the effect of a cyclical chemical equation is also (a), that is, the value of expressive substance of quantitative character is (a). Let the number of reaction factors of a cyclical chemical equation be $(\mathrm{m})$, i.e., in a cyclical chemical equation, the number of B, C, D...E, F, A is (m), and let the number of cyclical chemical equations for the synthesis of expressive substance of quantitative characters in an organism is ( $\mathrm{n})$, and it is equal to the number of genes controlling the expressive substance of quantitative characters. Then the total mass of a cyclical chemical equation is (ma), the total mass of (n) cyclical chemical equations is (nma). The number of reaction factors in a cyclical chemical equation is $(m)$, the number of reaction factors is $[n(m-1)+1]$ when $(n)$ cyclical chemical equations form a cyclic equilibrium system centering on the expressive substance (A).

\subsection{Calculation of the Mass of the Expressive Substance of Quantitative Characters}

In the context of the study of chemistry, the law of conservation of mass says, (Anne Marie Helmenstine, Ph.D., 2019), in a chemical reaction, the mass of the products equals the mass of the reactants. and the Le Châtelier's Principle says also, ( Jan W. Gooch, 2011), in a reaction that has achieved equilibrium, if one of the conditions affecting the equilibrium, such 


\section{IIMacrothink}

as temperature, pressure, and concentration of the chemicals involved in the reaction, is changed, the equilibrium will move in the direction that can weaken the change. Hence, in this cyclical equilibrium system, the total mass of all reactants is conservative, and the mass allocated to every reaction factor should also be equal, that is, the mass of the expressive substance (A) of quantitative character,

$$
\begin{gathered}
\mathrm{x}=\frac{n m a}{n(m-1)+1} \\
\mathrm{x} \geq 0, \quad \mathrm{a} \geq 0, \mathrm{n} \geq 1, \quad \mathrm{~m} \geq 2
\end{gathered}
$$

\subsection{Mathematical Elimination}

From

$$
\mathrm{x}=\frac{n m a}{n(m-1)+1}
$$

It can be obtained by conversion,

$$
\begin{gathered}
\frac{x}{a}-\frac{x}{m a}+\frac{x}{n m a}=1 \\
\because \frac{x}{n m a} \geq 0 \\
\therefore \frac{x}{a}-\frac{x}{a m} \leq 1 \quad \mathrm{x} \leq \frac{m}{m-1} \mathrm{a} \\
\because \mathrm{m} \geq 2 \quad \therefore \mathrm{x} \leq 2 \mathrm{a}
\end{gathered}
$$

That is to say, for a biological group, under the condition of normal growth and development of each individual, the maximum value of quantitative characters is less than or equal to two times of the minimum value.

\subsection{Application of the Equilibrium Law}

In order to facilitate our understanding, let us further analyze the equilibrium movement process of specific chemical reactions.

2.7.1 When $n=1$, there is only one cyclical chemical equation to synthesize the expressive substance of quantitative character (A) in cells, call it way ${ }_{1}$. As shown in Figure 1. 


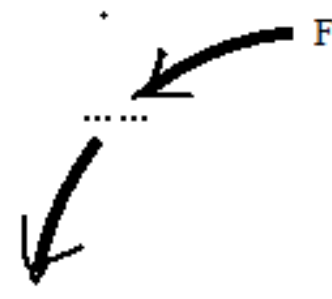

$B_{1}$

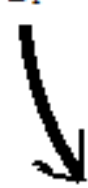

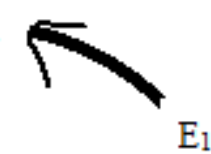

$E_{1}$

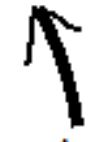

A
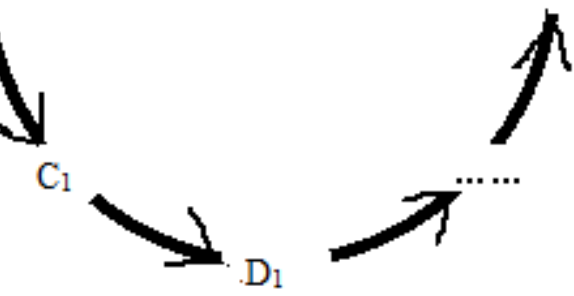

Figure 1 . When $n=1$, there is only one cyclical chemical equation to synthesize the expressive substance of quantitative character (A) in cells, call it way ${ }_{1}$

It means that the substance $B_{1}$ is transformed into the expressive substance (A) of quantitative character after a series of chemical changes, and $(\mathrm{A})$ is also transformed into $\mathrm{B}_{1}$ after a series of other chemical changes, the relationship between $\mathrm{B}_{1}$ and $(\mathrm{A})$ is a cyclical chemical equation that can be transformed into each other. In this cyclical chemical equation, if the mass of one factor is higher than other factors, according to the Le Chatelier's Principle (Jan W. Gooch, 2011), the extra mass will move along the direction of the cyclical chemical equation until the mass of each factor reaches the equilibrium.

The phenotype value of quantitative character,

$$
\mathrm{x}=\mathrm{a}
$$

2.7.2. When $n=2$, there are two cyclical chemical equations to synthesize quantitative characters expressive substance (A) in cells, as shown in Figure 2. 

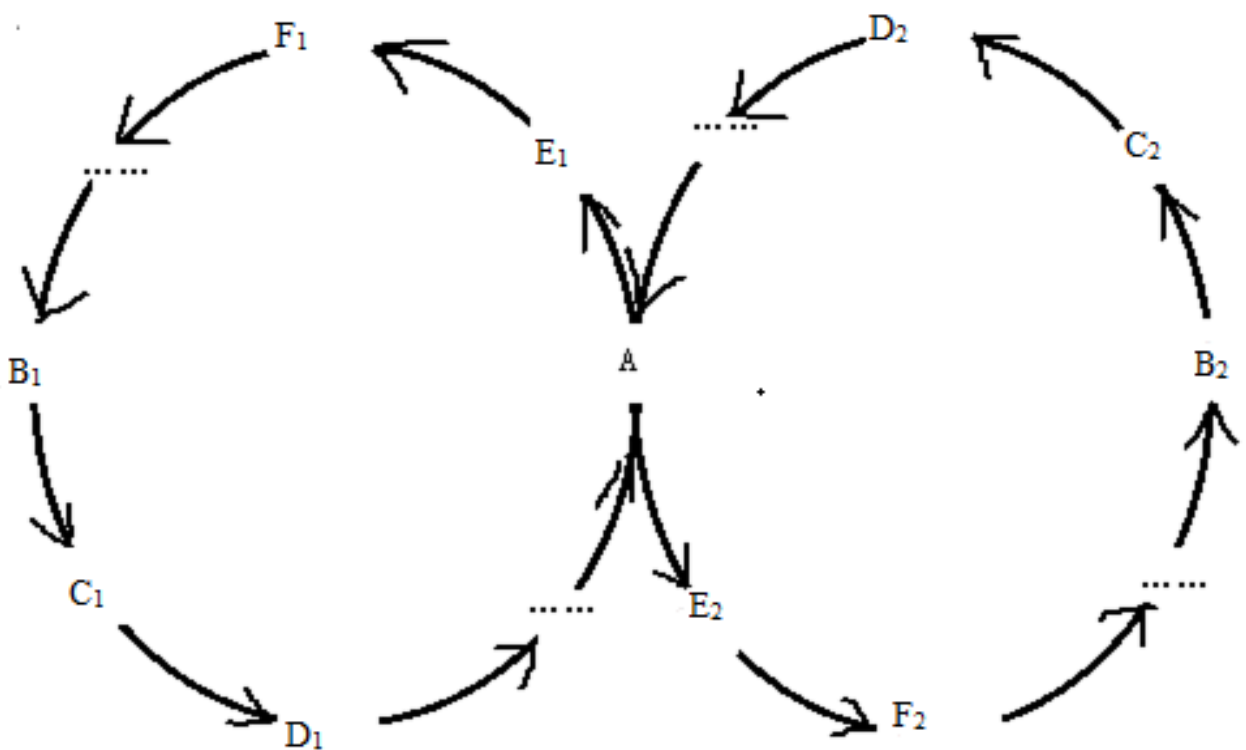

Figure 2. When $n=2$, there are two cyclical chemical equations to synthesize quantitative characters expressive substance (A) in cells

It means after a series of chemical changes, the substance $B_{1}$ is transformed into quantitative character expressive substance (A), and (A) is also transformed into B1 after a series of other chemical changes (way ${ }_{1}: \mathrm{B}_{1} \rightarrow \mathrm{C}_{1} \rightarrow \mathrm{D}_{1} \rightarrow \ldots \ldots \rightarrow \mathrm{A} \rightarrow \mathrm{E}_{1} \rightarrow \mathrm{F}_{1} \rightarrow \ldots \ldots \rightarrow \mathrm{B}_{1}$ ), at the same time, $B_{2}$ is transformed into expressive substance (A) and (A) is transformed into $B_{2}$, and it forms the way (way $_{2}: \mathrm{B}_{2} \rightarrow \mathrm{C}_{2} \rightarrow \mathrm{D}_{2} \rightarrow \ldots \ldots \rightarrow \mathrm{A} \rightarrow \mathrm{E}_{2} \rightarrow \mathrm{F}_{2} \rightarrow \ldots \ldots \rightarrow \mathrm{B}_{2}$ ), that is to say, there are two cyclical chemical equations to synthesize expressive substance (A) in cells. These two cyclical chemical equations take (A) as the center and form a circular equilibrium system $\left(\right.$ system $\left._{1}\right)$. In this system, due to the restriction of chemical reaction equilibrium factors, the mass of every factor is equal. Its total mass is $(2 \mathrm{ma})$, the total reaction factors number of the system is $[2(\mathrm{~m}-1)+1]$, the average mass of each factor is:

$$
\frac{2 m a}{2(m-1)=1}
$$

When the two cyclical chemical equations form a circular equilibrium system with (A) as the center, how do the masses of the factors in the circular equilibrium system achieves a balance? This process can ideally be divided into two steps:

In the first step, to distribute the phenotype value (a) of quantitative characters that is synthesized by way ${ }_{2}$ to each factor of way ${ }_{1}$. Hence, in this cyclical chemical equation, the 
mass of each factor of way ${ }_{1}$ is higher than the other factors of way ${ }_{2}$ (excluding A).

In the second step, way ${ }_{1}$ and way ${ }_{2}$ form a new chemical reaction system with (A) as the center, the mass of each factor in way ${ }_{1}$ is higher than that in way ${ }_{2}$, according to the equilibrium law ( Jan W. Gooch, 2011), the factors in way ${ }_{1}$ must have a certain mass of the expressive substances (A) to move into the factors in way ${ }_{2}$, in order to achieve a balance in the mass distribution of the two, until the mass of each factor of the two ways is equal.

Although the mass of the expressive substance (A) synthesized by single cyclical chemical equation, namely, way ${ }_{1}$ or way ${ }_{2}$ is (a), the effects produced by the two cyclical chemical equations are the same, however, when the way ${ }_{1}$ and way ${ }_{2}$ form a cyclic equilibrium system $\left(\right.$ system $\left._{1}\right)$, the effect of system ${ }_{1}$ is not a simple addition of the effects of way and way $_{2}$, i.e., (2a), it is because each cyclical chemical equation is composed of multiple reaction factors, the increased effect of way ${ }_{2}$ is evenly distributed by these reaction factors, it is also the root cause of the micro effect of quantitative character's control gene.

2.7.3. When $n=3$, there are three ways to synthesize expressive substance(A) in cells. As shown in Figure 3,

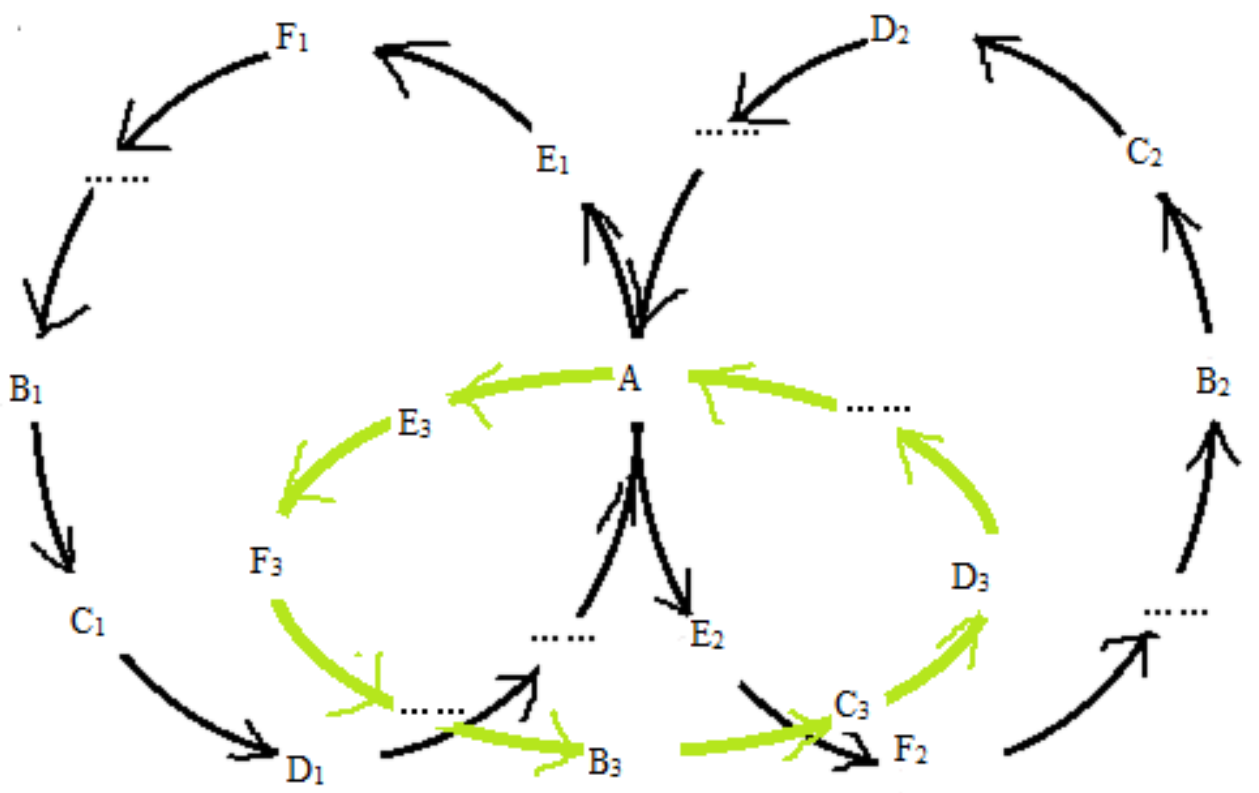

Figure 3 . When $n=3$, there are three ways to synthesize expressive substance(A) in cells 


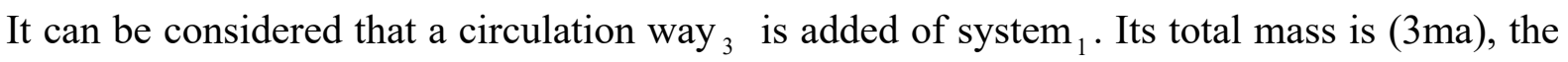
total number of reaction factors of the system is $[3(\mathrm{~m}-1)+1]$, the average mass of each factor is:

$$
\frac{3 m a}{3(m-1)=1}
$$

The process of balancing the mass of each factor in the system can also be idealized into two steps:

In the first step, the expressive substance (A) synthesized in way ${ }_{3}$ was evenly assigned to each factor of system ${ }_{1}$. In this way, the mass of each factor of system ${ }_{1}$ is higher than the other factors of way $_{3}$ (excluding A).

Second, because of the chemical reaction equilibrium, the extra mass of expressive substance (A) in each factor of system ${ }_{1}$ were transferred to each factor of way $_{3}$, in order to achieve a balance of the distribution of mass, until the mass of each factor in the newly generated system $\left(\right.$ system $\left._{2}\right)$ is equal.

\subsubsection{When (n) Is Infinite}

By analogy, it will construct a complex cyclical equilibrium system to synthesize the expressive substance (A). No matter how complex the system is, it should follow the law of conservation of mass and the equilibrium law. Therefore, the effect of quantitative character's genes are not simply cumulative, it is restricted by chemical equilibrium factors in the process of chemical reaction. No matter how many genes in an organism to control quantitative character's expression, their combined effect is less or equal than twice that of a single gene.

In the above analysis, (a) can be regarded as the average value of the effect of each cyclical chemical equation, (m) can be regarded as the an average of the number of reaction factors of all cyclical chemical equations.

\section{Results and Discussion}

\subsection{Results}

The inheritance of quantitative characters is controlled by many genes, however, the expression of quantitative characters can only be realized on specific chemical reactions. In the specific chemical reaction process of quantitative character expression, the law of conservation of mass and the equilibrium law is the two objective laws that must be followed. 


\section{Macrothink}

\subsection{Discussion}

\subsubsection{The Application of the Law of Conservation of Mass in Organic Chemistry}

For a long time, due to the multi-directionality and complexity of chemical reactions, the application of the law of conservation of mass in organic chemistry is a difficult problem, the idea of a cyclical equilibrium system of chemical reactions provides a useful attempt to solve this problem.

\subsubsection{There Are Two Reaction Centers in a Cyclical Equilibrium System of Chemical} Reactions

When there are two or even more reaction centers in a cyclical equilibrium system of chemical reactions, it does not affect the application of the law of conservation of mass and the equilibrium law in this reaction system, and the effect is exactly the same as that has a reaction center only. As shown in Figure 4.
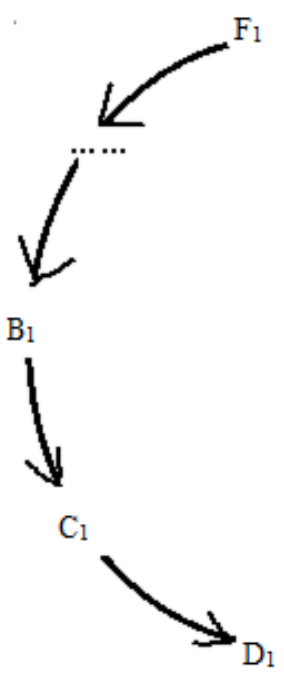
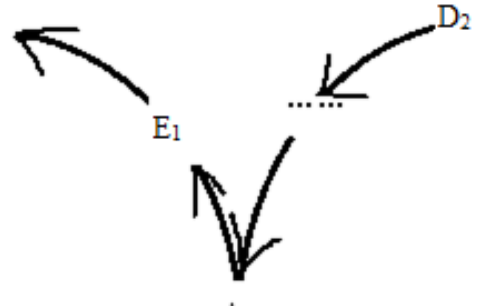

A

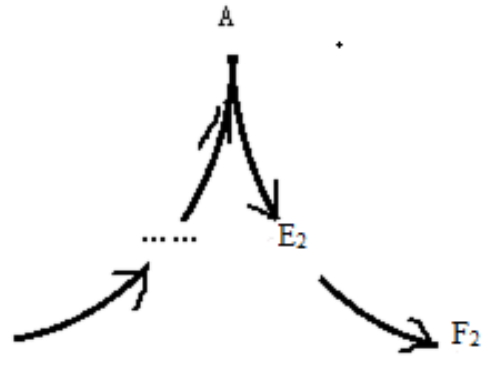

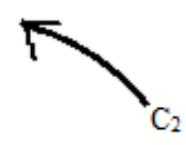
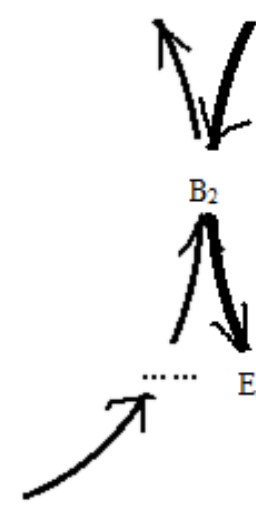

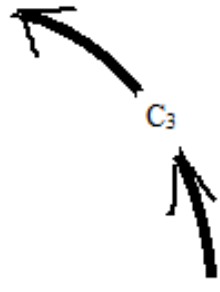

$\mathrm{B}_{3}$

Figure 4. The cyclical equilibrium system of chemical reactions

The cyclical equilibrium system of chemical reactions shown in Figure 4 has the same effect as that shown in Figure 3.

\section{Conclusion}

The inheritance of biological traits is controlled by genetic material, however, the expression of biological traits must be realized on some specific chemical reactions. The various chemical reactions in an organism do not occur in disorder, instead, they must follow some objective laws, the law of conservation of mass and the equilibrium law is the embodiment of these objective law. 


\section{References}

Anne Marie Helmenstine, Ph.D., Law of Conservation of Mass, Thought Co. (science). https://www.thoughtco.com/definition-of-conservation-of-mass-law-604412

Gooch, J. W. (2011). Le Châtelier's Principle, Encyclopedic Dictionary of Polymers, 424-424. https://doi.org/10.1007/978-1-4419-6247-8_6859

Liu, Q. C. (2009). Genetics (Second Edition): Science Press.

\section{Copyright Disclaimer}

Copyright for this article is retained by the author(s), with first publication rights granted to the journal.

This is an open-access article distributed under the terms and conditions of the Creative Commons Attribution license (http://creativecommons.org/licenses/by/4.0/). 